\title{
Rhabdomyolysis in Acute Spinal Cord Injury Presenting With Nausea and Vomiting as Chief Complaints: A Case Report
}

\author{
Hae Rim Kim, MD, Hyun Mi Oh, MD, A Sa Yeon Choi, MD, Jong In Lee, MD
}

Department of Rehabilitation Medicine, The Catholic University of Korea College of Medicine, Seoul, Korea

Dealing with complications is crucial in the management of patients with spinal cord injury (SCI). We describe a case of rhabdomyolysis in SCI without apparent soft tissue injury, presenting with nausea and vomiting as chief complaints. Given that gastrointestinal discomfort is common in SCI, this case highlights the need to consider rhabdomyolysis as a potential cause of unexplained nausea and vomiting in SCI, and indicate the value of regular check-up of creatine kinase level in SCI patients. Early diagnosis and treatment can prevent acute renal failure that can occur with rhabdomyolysis and minimize the potential threat of declined renal function in SCI patients.

Keywords Spinal cord injuries, Rhabdomyolysis, Nausea

\section{INTRODUCTION}

Rhabdomyolysis is a syndrome caused by skeletal muscle injury that involves leakage of large quantities of potentially toxic intracellular contents into plasma, which can cause renal failure [1]. It is characterized by the triad of muscle weakness, myalgia, and dark urine [2]. Rhabdomyolysis is relatively common in multiple trauma, and should be suspected when there is apparent soft tissue injury or muscular symptoms associated with renal involvement. In contrast, less severe forms of rhabdomy-

Received August 1, 2013; Accepted September 2, 2013

Corresponding author: Jong In Lee

Department of Rehabilitation Medicine, Seoul St. Mary's Hospital, The Catholic University of Korea College of Medicine, 222 Banpo-daero, Seocho-gu, Seoul 137-701, Korea

Tel: +82-2-2258-2822, Fax: +82-2-2258-2825, E-mail: ljikyh@catholic.ac.kr

(c) This is an open-access article distributed under the terms of the Creative Commons Attribution Non-Commercial License (http://creativecommons. org/licenses/by-nc/3.0) which permits unrestricted noncommercial use, distribution, and reproduction in any medium, provided the original work is properly cited.

Copyright (c) 2014 by Korean Academy of Rehabilitation Medicine olysis may not cause any muscular symptoms, and the diagnosis is based on laboratory studies in the context of other problems $[3,4]$. Here, we describe an unusual case of rhabdomyolysis in an acute spinal cord injury (SCI) patient without apparent muscular injury, presenting with nausea and vomiting as chief complaints.

\section{CASE REPORT}

A 34-year-old female patient fell from a 10-m height and was immediately moved to an adjacent emergency center. Thoracic spine fracture (T12) with retropulsion into the spinal canal causing T12 myelopathy was diagnosed. Mega-dose steroid therapy was started, followed by transfer to the Department of Neurosurgery in our hospital. She underwent decompression of the spinal canal followed by posterior fusion of the spine. The procedure took 4 hours. After postoperative stabilization, the patient was referred to the Department of Rehabilitation Medicine for further treatment. On clinical examina- 
tion, her cardiopulmonary system was stable and she was a complete paraplegic (the American Spinal Injury Association [ASIA] grade A, neurological level of injury L1). She had well-controlled schizophrenia for 10 years, without any positive symptoms including hallucination. She had taken $400 \mathrm{mg}$ clozapine, $1 \mathrm{mg}$ benzatropine, and $40 \mathrm{mg}$ ziprasidone every day for several years without significant side-effects. No other systemic illnesses or family histories were noted. She did not smoke or abuse drugs, and was not dependent on alcohol. On initial evaluations including whole body bone scan, there were no other combined injuries. After being transferred to the rehabilitation department, she started an active rehabilitation program.

About 1 week after beginning active rehabilitation, she complained of intermittent nausea and vomiting without abdominal pain, myalgia, or other systemic symptoms. The symptoms persisted even after management of fecal impaction. She did not have past history of gastrointestinal problems, and physical examination did not reveal abdominal tenderness or other positive findings indicative of gastrointestinal lesions. Abdominal X-ray did not show any specific abnormalities. The findings lessened the possibility of gastritis or ulcerative lesions, so gastric fiberscopy was not done. Blood chemistry did not show any evidence of uremia or electrolyte imbalance. Her drugs were reviewed for possible emetic effect and administration of pregabalin was stopped, but the symptoms did not improve. Brain magnetic resonance imaging taken on admission was unremarkable and, since she did not complain of headache, intracranial lesion was ruled out. According to the neuropsychiatric consultation report, neuroleptic malignant syndrome was unlikely, since she had taken the same antipsychotics for several years without other related symptoms or signs of the syndrome.

One week after the onset of gastrointestinal symptoms, she presented with tea-colored urine. Rechecked blood chemistry revealed elevated creatine kinase (CK, 1,797 $\mathrm{IU} / \mathrm{L}$ ) and lactate dehydrogenase (459 IU/L); these levels had been normal on the day of admission. The subtype of CK was MM, and the serum levels of other cardiac enzymes were normal. Other laboratory finding were as follows: alanine transaminase $35 \mathrm{IU} / \mathrm{L}$, aspartate transaminase $66 \mathrm{IU} / \mathrm{L}$, blood urea nitrogen $7.3 \mathrm{mg} / \mathrm{dL}$, creatinine $0.58 \mathrm{mg} / \mathrm{dL}$, potassium $4.4 \mathrm{mEq} / \mathrm{L}$, phosphorus 4.8 $\mathrm{mg} / \mathrm{dL}$, and alkaline phosphatase $59 \mathrm{IU} / \mathrm{L}$. Urine level of myoglobin was elevated, exceeding $1,000 \mathrm{ng} / \mathrm{mL}$. Whole body bone scan was done again; no soft tissue injury and development of heterotrophic ossification were evident.

The elevated CK level of muscle origin over five times the upper limit of normal and associated myoglobinuria fit the diagnostic criteria of rhabdomyolysis. She was managed with intravenous saline treatment and her physical activities were reduced to prevent acute renal failure, with close monitoring of electrolyte and renal function. After starting hydration, urine color was normalized and nausea and vomiting subsided. After 2 weeks of hydration, CK level was normalized without development of renal failure throughout the period (Fig. 1).

\section{DISCUSSION}

SCI patients frequently complain of nausea and vomiting. The causes vary. High cervical SCI is commonly combined with gastric ulcer. Prolonged immobility and impaired bowel function frequently lead to constipation. Many drugs can cause gastritis or the drug itself can have an emetic effect. Electrolyte imbalance and uremia can develop as consequences of bladder dysfunction. Brain injury, commonly combined in cases of traumatic SCI, can cause emesis. However, as far as is known, there is no reported case of SCI with rhabdomyolysis whose main

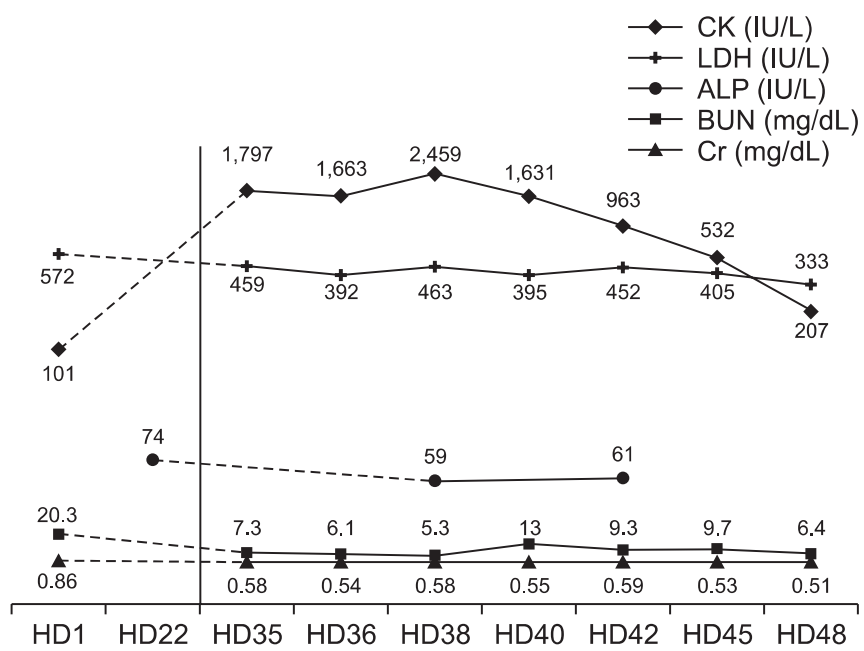

Fig. 1. Serial level of blood chemistry. Rhabdomyolysis was diagnosed on hospital day (HD) 35. CK, creatine kinase; LDH, lactate dehydrogenase; ALP, alkaline phosphatase; BUN, blood urea nitrogen; $\mathrm{Cr}$, creatinine. 
problems were nausea and vomiting, as reported in this paper.

This patient did not present any typical symptoms or signs of rhabdomyolysis. Initially there was no evidence of muscular breakdown. The whole body bone scan, a valuable tool for screening actual muscular damage $[5,6]$, was obtained twice, but no muscular injuries were detected. She did not complain of myalgia throughout the entire hospitalization. On physical examination, there was no evidence of soft tissue injury below the patient's sensory level. Intermittent nausea and vomiting were the primary clues before the typical tea-colored urine appeared.

The cause of rhabdomyolysis in this case can be explained by mild immobilization resulting in subclinical compressive ischemia of muscle [7]. Rhabdomyolysis can be caused by non-physical causes like metabolic myopathies, drugs and toxins, infections, electrolyte abnormalities, endocrine disorders, or polymyositis/ dermatomyositis. However, these were ruled out in this case, since there were no electrolyte imbalance, signs of viral infection, recurrent myoglobinuria, exercise intolerance, or other relevant past histories $[8,9]$. Several aspects can be suspected as the cause of immobilizationrelated muscle ischemia. The surgery was prolonged and the paraplegia limited physical activities significantly. Insensibility of SCI patients to pain caused by soft tissue injury under the sensory level suggests the possibility of subclinical compression of muscles. To our knowledge, there are no reported data studying the time between the event of immobilization and the onset of rhabdomyolysis. However, a similar case of SCI was reported in 1995, which was complicated by pressure-induced rhabdomyolysis of delayed onset. It was associated with unnoticed events of subclinical muscle compression, resulting in renal dysfunction [7]. It was hypothesized that all of the subclinical events had cumulatively contributed to cause the delayed-onset of rhabdomyolysis. The delay was interpreted as the result of beginning of physical activities causing the products of muscle catabolism to flush into the bloodstream [7]. Our case can be interpreted in similar way, since rhabdomyolysis developed 1 week after beginning active rehabilitation.

This case differs from the previously reported case, in that the chief complaints were nausea and vomiting, and the renal failure did not develop. Rhabdomyolysis is a po- tentially fatal disease with complications like acute renal failure [8]. If clinicians diagnose it earlier, renal function can be preserved by intensive hydration. However, rhabdomyolysis without typical symptoms or noticeable history of trauma is not easily recognized. It can even be asymptomatic, or the main symptoms can be non-specific like the gastrointestinal discomfort of this case. Since this case was not complicated with renal involvement at the time of diagnosis, we could prevent acute renal failure with intensive hydration.

After experiencing this unexpected case of rhabdomyolysis in SCI heralded by nausea and vomiting, we recommend regularly check of CK level and consideration of rhabdomyolysis as a potential cause of unexplained nausea and vomiting. In this way, rhabdomyolysis will be diagnosed earlier and renal function can be preserved, which is one of the main concerns in managing SCI patients.

\section{CONFLICT OF INTEREST}

No potential conflict of interest relevant to this article was reported.

\section{REFERENCES}

1. Beetham R. Biochemical investigation of suspected rhabdomyolysis. Ann Clin Biochem 2000;37 (Pt 5):581-7.

2. Cervellin G, Comelli I, Lippi G. Rhabdomyolysis: historical background, clinical, diagnostic and therapeutic features. Clin Chem Lab Med 2010;48:749-56.

3. Huerta-Alardin AL, Varon J, Marik PE. Bench-to-bedside review: Rhabdomyolysis: an overview for clinicians. Crit Care 2005;9:158-69.

4. Bosch X, Poch E, Grau JM. Rhabdomyolysis and acute kidney injury. N Engl J Med 2009;361:62-72.

5. Fleckenstein JL, Chason DP, Bonte FJ, Parkey RW, Hunt JL, Purdue GF, et al. High-voltage electric injury: assessment of muscle viability with MR imaging and Tc-99m pyrophosphate scintigraphy. Radiology 1995;195:205-10.

6. Park JM, Shin HJ, Choi YC. Acute rhabdomyolysis: importance of MRI and bone scintigraphy. J Korean Soc Pediatr Nephrol 2009;13:92-5.

7. Prall JA, Breeze RE. Rhabdomyolysis following spinal 
Hae Rim Kim, et al.

cord injury: case report. J Trauma 1995;39:802-4.

8. Vanholder R, Sever MS, Erek E, Lameire N. Rhabdomyolysis. J Am Soc Nephrol 2000;11:1553-61.
9. Darras BT, Friedman NR. Metabolic myopathies: a clinical approach, part I. Pediatr Neurol 2000;22:8797. 